\title{
SAMI - A new multi-object IFS for the Anglo-Australian Telescope
}

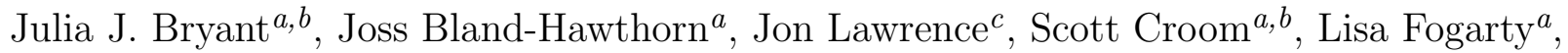 \\ Michael Goodwin ${ }^{c}$, Samuel Richards ${ }^{a}$, Tony Farrell ${ }^{c}$, Stan Miziarski ${ }^{c}$, Ron Heald ${ }^{c}$, Heath \\ Jones ${ }^{d}$, Steve Lee ${ }^{c}$, Matthew Colless ${ }^{c}$, Michael Birchall ${ }^{c}$, Andrew M. Hopkins ${ }^{c}$, Sarah Brough ${ }^{c}$ \\ Amanda E. Bauer ${ }^{c}$ \\ ${ }^{a}$ Sydney Institute for Astronomy (SIfA), School of Physics, The University of Sydney, NSW, \\ Australia 2006; \\ ${ }^{b}$ ARC Centre of Excellence for All-sky Astrophysics (CAASTRO); \\ ${ }^{c}$ Australian Astronomical Observatory, P.O. Box 296, Epping, NSW, Australia 1710; \\ ${ }^{d}$ School of Physics, Monash University, Clayton, VIC 3800, Australia.
}

\begin{abstract}
SAMI (Sydney-AAO Multi-object Integral field spectrograph) has the potential to revolutionise our understanding of galaxies, with spatially-resolved spectroscopy of large numbers of targets. It is the first on-sky application of innovative photonic imaging bundles called hexabundles, which will remove the aperture effects that have biased previous single-fibre multi-object astronomical surveys. The hexabundles have lightly-fused circular multi-mode cores with a covering fraction of $\sim 73 \%$. The thirteen hexabundles in SAMI, each have 61 fibre cores, and feed into the AAOmega spectrograph at the Anglo-Australian Telescope (AAT). SAMI was installed at the AAT in July 2011 and the first commissioning results prove the effectiveness of hexabundles on sky. A galaxy survey of several thousand galaxies to $z \sim 0.1$ will begin with SAMI in mid-2012.
\end{abstract}

Keywords: Optical fibre; hexabundles; astronomical instrumentation; galaxy surveys

\section{INTRODUCTION}

Spectroscopic galaxy surveys using single fibres or single slits have amassed spectra of hundreds of thousand of galaxies ${ }^{1,9,12,18}$. A wide range of galaxy properties have been deduced from these large statistical samples, including stellar ages, star formation rates and metallicites, black hole accretion ratios and extinction. However, the limitation on such surveys is that all galaxies are sampled by the same size slit or fibre, irrespective of the galaxy size, morphology or distance. Therefore only a snapshot of one part of the galaxy is observed, and it is a different fraction of the galaxy in each case. This can lead to biases in the global conclusions from large samples.

Spatially-resolved spectroscopy, or integral field spectroscopy (IFS) alleviates these biases and gives resolved spectroscopy across each galaxy. By sampling the spectra over many spatial elements across a galaxy it becomes clear how much of the galaxy (by fitting) is observed in each case. The distribution of star formation, stellar ages and dynamical mass can be measured, along with the dynamics associated with mergers and interactions. The success of IFS has been shown with small samples of galaxies (using e.g. SAURON ${ }^{2}$ and SINFONI $^{20}$ ). The next step is to achieve IFS on large samples of thousands of galaxies, to disentangle these properties over a vast range of environments, redshifts and galaxy types.

Over the last 2 years, the development of a new multi-fibre imaging bundle technology called hexabundles ${ }^{4-6}$ has resulted in an imaging device that can be replicated many times in a given field and positioned in a similar way to that used for single-fibre survey instruments in the past. The first on-sky test of hexabundles is through a new demonstrator instrument called the Sydney-AAO Multi-object Integral field spectrograph (SAMI $\left.{ }^{11}\right)$ which is installed on the Anglo-Australian Telescope (AAT) at Siding Spring Observatory, NSW, Australia. SAMI allows simultaneous observations of 13 galaxies with hexabundles positioned across a 1 degree on-sky field. SAMI has successfully proven this new technology, and is now having an upgrade of the bundles and fibre run to use a fibre

J.J.B.: E-mail: jbryant@physics.usyd.edu.au

Ground-based and Airborne Instrumentation for Astronpmy IV, edited by lan S. McLean, Suzanne K. Ramsay, Hideki Takami, Proc. of SPIE Vol. 8446, 84460X · (c) 2012 SPIE · CCC code: 0277-786X/12/\$18 - doi: 10.1117/12.925115 
with higher throughput. A major galaxy survey with SAMI is beginning in 2012B. Plans are already underway for a very much larger instrument called HECTOR ${ }^{17}$ which will have 50-100 hexabundles over a 2-3 degree field.

In Section 2, I will give a summary of the key science motivating SAMI, followed by a description of the instrument design in section 3. Early galaxy observations with SAMI are shown in section 4 .

\section{SCIENCE MOTIVATION}

The key science that can be achieved with an IFS galaxy survey, and in particular, with SAMI, has been discussed in detail in Croom et al. (2012). The main science objectives for SAMI include:

- Star formation in galaxies: In colour-magnitude space, galaxies roughly divide between those that are on the blue sequence and have significant star formation, and those on the red sequence that have much less star formation and typically live in denser environments ${ }^{8,19}$. Proposed mechanisms by which a galaxy on the blue sequence can lose gas in order to quench star formation and move to the red sequence include ram pressure stripping resulting from falling into a dense cluster medium, then strangulation, where the galaxy gas reservoir is depleted until no more stars are formed and the existing population ages, reddening the galaxy $^{16}$. However, the interactions between galaxies have also been proposed to trigger star formation ${ }^{13}$ - Resolving the current star formation distribution using emission lines, the past star formation history using stellar ages and metallicities and coupling that to the dynamics with IFS from SAMI, can directly address the evolution of star formation in galaxies over time. The separation of disk and bulge components of a galaxy is possible with SAMI and will then allow the evolution of each component to be disentangled.

- The build up of stellar mass in a galaxy is directly related to the feeding of a central super-massive black hole $^{24}$. However, the exact connection between the Active Galactic Nucleus (AGN) and star formation activity is unclear. There is some evidence that mergers and interactions between galaxies can trigger star formation or AGN activity ${ }^{15}$, but at the same time, the jets from an AGN can trigger star formation independently. Spatially resolving the star forming regions in galaxies of different masses can identify direct correlations between star forming regions and the effect of the AGN. Emission line and absorption line velocities provide gas and stellar kinematics which in turn give dynamical galaxy mass estimates.

- Gas accretion in galaxies: The mechanisms by which gas enters a galaxy are still unknown ${ }^{3,7,21}$. Outer warps in galaxy disks can be detected in ionized gas. SAMI has the potential to resolve the kinematics of gas in the outer disk and relate that to galaxy activity (including AGN activity and jets) and dynamical disturbances. A large SAMI survey is likely to detect an statistically significant sample of galaxies with warm gas extending vertically off the plane of the galaxy, ${ }^{14}$ and this can then be understood by comparison with morphology, mass, star formation distribution, and nuclear activity.

\section{SAMI INSTRUMENT DESIGN}

\subsection{COMPONENTS OF SAMI}

SAMI is installed at the prime-focus top end of the $3.9 \mathrm{~m}$ Anglo-Australian Telescope (AAT). Thirteen 61-core hexabundles and 26 sky fibres, are mounted onto a plug-plate (see Figure 1) in the triplet corrector top-end focus of the AAT, which has a 1-degree field of view. The original SAMI prototype used for the galaxy observations shown below had each hexabundle mounted in an SMA connector, however, the upgraded SAMI has replaced the SMA connectors with custom magnetic connectors. A stress-relieving sleeve and reinforced flexible plastic tubing support the fibres coming away from the plate. The positioning and repositioning of the SMA connector for each field configuration is precise to less than half a core diameter. Mounting the hexabundles directly at the prime focus, minimises extra optical surfaces and eliminates the need for lenslets or microlens arrays. The $\mathrm{f} / 3.4$ input ensures minimal focal ratio degradation (FRD) from the fibres (see section 3.2.2 below). Each of the 61 cores has an effective view of 1.6 arcsec, and the total diameter of each bundle is 15 arcsec.

The 61 fibres coming from each of the 13 hexabundles then feed into a splice box, where they are spliced to $42 \mathrm{~m}$ lengths of fibre. This allows the fibre to then travel from the top end of the telescope, to the coudé room on the telescope floor, where the AAOmega spectrograph ${ }^{22}$ is located. The fibres are terminated in glass 

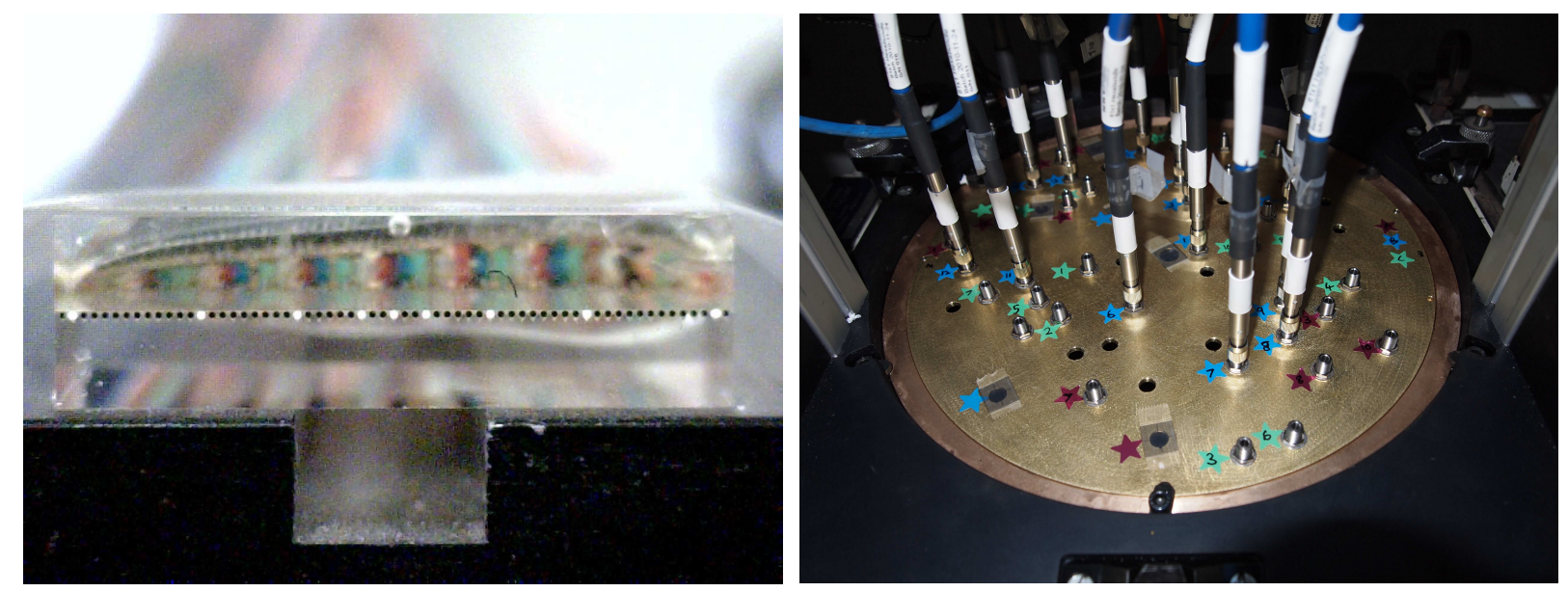

Figure 1. Left Close-up view of the front of one slit block that feeds into the slit of the AAOmega spectrograph. The individual fibres from a hexabundle are lined up in v-grooves and secured in the glass block. In this view, nine cores are illuminated. Right SAMI hexabundles, installed in the prime focus top end of the AAT. The hexabundles are mounted inside metal ferules with SMA attachments, and are shown here plugged into the field plate. The protective blue tube then leads to the splice box.

slit blocks as shown in Figure 1, one for each hexabundle plus 2 sky fibres. These slit blocks are then mounted with index matching gel, against the lens that forms the slit into AAOmega. AAOmega is a dual-beam fibre spectrograph with volume-phase holographic $(\mathrm{VPH})$ gratings giving resolutions of $\sim 1700$ to $\sim 13000$. The number of hexabundles was set by the space on the AAOmega slit, allowing also for two sky fibres per 61 hexabundles cores.

The fibre type used in the hexabundles and the fibre run, is AFS105/125Y fibre, with $105 \mu \mathrm{m}$ core and $125 \mu \mathrm{m}$ cladding. However the resultant throughput in the blue is rather poor and therefore this fibre is being replace with a fibre that has much better throughput (see section 3.2 .2 below). The original $42 \mathrm{~m}$ fibre cable was ribbonised to protect the fibres, however we have found that the FRD resulting from ribbonised fibres is very large $\mathrm{e}^{11}$ and as a result, the new replacement fibres will have a different protective packaging. Several options are being tested for the outer tube, and both acrylate and tefzel buffers have been considered for each fibre. While the original SAMI produced excellent galaxy images and spectra as shown in Section 4 below, the upgraded SAMI will have a significant boost in throughput and performance.

\subsection{HEXABUNDLES AND THEIR PERFORMANCE}

Hexabundles ${ }^{4-6}$ are imaging fibre bundles which can take spatially-resolved spectra of many objects across a field and can be positioned similarly to single-fibres in a multi-object spectroscopic (MOS) instrument. Each of the SAMI hexabundles consists of 61 optical fibres, each with a $105 \mu \mathrm{m}$ core and a numerical aperture (NA) of 0.22 , as shown in Figure 2. The cladding is reduced down to only $\sim 5 \mu \mathrm{m}$ thick for a few centimetres at the input end, to minimise cross-talk, and the fibres are then fused to form a bundle. Key new advances in this technology ensure that now a fibre bundle can be made, which can maintain a high filling factor of $\sim 73 \%$, but with significantly lower FRD than previous fused bundles. After the short fused region, the 61 fibres are separate fibre pigtails.

\subsubsection{FRD AND THROUGHPUT TESTING METHOD}

Detailed FRD, throughput and cross-talk testing has been done for hexabundles, with a range of input beam speeds in Bryant et al. (2012). Details discussed here are for the SAMI case in which, the input speed is $\mathrm{f} / 3.4$ into the hexabundles, and the acceptance cone of the spectrograph is $\mathrm{f} / 3.15$. The testing setup for the hexabundles has an LED light source that is fed through a filter holder then into a fibre which is then re-imaged to form an $\mathrm{f} / 3.4$ beam. Each measurement is done through each of two Bessel filters; a B-band filter centred on $457 \mathrm{~nm}$ with a FWHM of $27 \mathrm{~nm}$ and an R-band filter centred on $\sim 596 \mathrm{~nm}$ with an asymmetric profile of $60 \mathrm{~nm}$ 


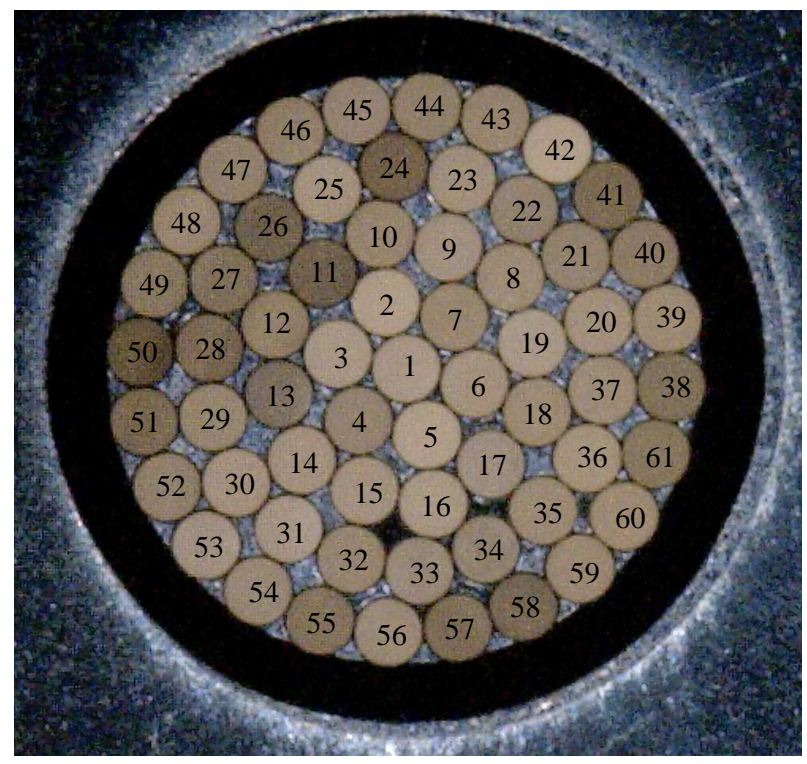

Figure 2. Front face of one of the 61-core fused hexabundles used in the SAMI instrument. The cores are $105 \mu \mathrm{m}$ in diameter, or 1.6 arcsec per fibre, giving 15 arcsecs across each hexabundle. The fibres were back-illuminated randomly.

width. The focussed $\mathrm{f} / 3.4$ input spot is $<50 \mu \mathrm{m}$ by $<0.5 \%$ intensity and therefore smaller than the core size to ensure no light is input into adjacent cores to contaminate cross-talk measurements. The input was focussed onto individual bare fibres and a sample of cores in each hexabundle tested in turn. Cores were chosen to be at different radial positions throughout the bundle.

The output individual fibre pigtails were $\sim 3 \mathrm{~m}$ long. The ends were carefully cleaved and checked under a microscope to ensure the cleave crack did not penetrate the core, the cleave was straight to within $<<1$ degree, and the surface structure was minimised to a predetermined level, set at the point where surface structure variations result in measurement differences that are much less than other experimental errors (for details, $\operatorname{see}^{5}$ ). Cleaved ends were then mounted on a v-groove stage that was aligned to the axis of a pair of camera lenses that re-imaged the output onto an SBIG camera set at a precise back focus position. The input beam was characterised using the same imaging system. The output images were processed using scripts based on IRAF ${ }^{25}$ - First the images were dark subtracted, then the barycentre of each output profile was fitted and aperture photometry then gave the encircled energy with radius. From the back focal distance and pixel size, the output NA vs encircled energy was measured. Total integrated counts could then also be compared between the core with the input light, and the surrounding adjacent cores, to assess cross-talk.

Any test set-up to measure FRD can introduce artificial FRD due to misalignments or design. Full details of the tolerances of every component of our setup, and the resultant errors are analysed in detail in Bryant et al. (2012), including the effects of the end finishing.

The method for measuring throughput was to first find the total counts out of a $13 \mathrm{~m}$ bare AFS fibre. Counts were measured using aperture photometry in IRAF. Measured values are total counts, not counts within the $\mathrm{f} / 3.15$ output (fed into AAOmega) and hence are independent of FRD as such. The $13 \mathrm{~m}$ of AFS fibre was cut and spliced together several times, giving variations in counts of up to $2.8 \%$ for the worst splice. The splice was cut and a $40 \mathrm{~m}$ section of the AFS105/125Y fibre and the new SAMI fibre were each spliced in in turn, and counts measured. The same was repeated for several pieces of the new SAMI fibre type, with both Tefzel and Acrylate coating. By this cut-back method, the input coupling and the end face losses are accounted for.

\subsubsection{FRD AND THROUGHPUT RESULTS}

Focal ratio degradation is the result of imperfections in a fibre, microbends, and treatment or handling of the fibre. FRD results in a larger output light cone than that put in. Extensive work has been done to not only 
Table 1. Throughput of the central core of an original SAMI hexabundle with a fibre pigtail of $\sim 3 \mathrm{~m}$. The AFS105/125Y fibre used in SAMI (for the hexabundles and the fibre run), is then compared to the new replacement fibre, in the $B$-band and $R$-band. The replacement fibre will improve the throughput of both the hexabundles and the fibre run. The length of fibre from the hexabundles to the slit blocks that feed into AAOmega, is $42 \mathrm{~m}$. The errors are a combination of the light source variability, measurement errors, variation in the splices and the variation in results between different samples of fibre. These percentages have not been corrected for the losses in the 2 splices, which would typically increase these throughput by $1-2 \%$.

\begin{tabular}{lcc}
\hline Fibre & \multicolumn{2}{c}{$\%$ Throughput } \\
Type & $R$-band & $B$-band \\
& $\sim 596 \mu \mathrm{m}$ & $\sim 457 \mu \mathrm{m}$ \\
\hline \hline Central core of a hexabundle alone & $96 \pm 6$ & $89 \pm 5$ \\
$42 \mathrm{~m}$ bare AFS105/125Y & $78 \pm 5$ & $58 \pm 5$ \\
$42 \mathrm{~m}$ new replacement SAMI fibre & $91 \pm 3$ & $82 \pm 3$ \\
\hline
\end{tabular}

reduce FRD in the fibre run itself through stress relief and careful handling, but in particular, the manufacture of the hexabundles has been considerably refined to the point where the FRD is not significantly different to that of the bare fibre as shown in Figure 3.

The current SAMI hexabundles have an $\mathrm{f} / 3.4$ input, but the output into the AAOmega spectrograph is at $\mathrm{f} / 3.15$. Therefore the fraction of encircled energy into AAOmega is $92-95 \%$ in the red and $89-93 \%$ in the blue. These values reflect FRD, but not fibre throughputs. The new replacement SAMI hexabundles will be at least this good, and will use a fibre with a much higher blue throughput. The throughput for the $42 \mathrm{~m}$ fibre cable with the new fibre, compared to the original fibre used in SAMI is compared in Table 1. Most significantly, the new fibre type will improve the throughput of the SAMI fibre run by $\sim 13 \%$ in the $R$-band and $\sim 24 \%$ in the $B$-band.

Cross-talk from an illuminated central core into all of the surrounding touching cores was found to be $<0.5 \%$, which is insignificant compared to the effect of the seeing.

\section{SAMI FIRST ONSKY GALAXY DATA}

The first commissioning data for SAMI was taken in July 2011 and included a sample of galaxies at $z \leq 0.05$. Using the $580 \mathrm{~V}$ grating in the blue arm of AAOmega, we had a wavelength coverage of $370-570 \mathrm{~nm}$ and a resolution of $R=1730$. In the red arm, the $100 \mathrm{R}$ grating gave $625-735 \mathrm{~nm}$ at a resolution of $R=4500$. Full details of the observations, reduction and calibration tests for SAMI are given in Croom et al. (2012), however, here we discuss a summary of SAMI's on-sky performance and new dithering results.

\subsection{SAMI Data Reduction}

The SAMI data reduction is performed by $2 \mathrm{dfdr}^{10,23}$, a robust reduction pipeline previously developed for AAOmega and SPIRAL data and expanded to process SAMI data. The raw frames are bias subtracted and flat-fielded before each of the 819 spectra are extracted via an optimised algorithm. All extracted spectra are then wavelength calibrated, sky subtracted and corrected for relative fibre-to-fibre throughput variations. The reduced spectra are then reformatted into an $819 \times 2048$ pixel row-stacked spectrum (RSS) file. These RSS files are the output from $2 \mathrm{dfdr}$.

For the commissioning run in July 2011 one galaxy field containing 13 targets was observed. Two observing strategies were used for comparison, namely pointed observing, where the telescope was not moved between frames and dithered observing with small $(\sim 1 / 2$ core $)$ offsets applied between frames. The aim of the latter strategy is to fill in the gaps in the non-contiguous field of view of the hexabundles and to increase the spatial sampling of the data.

For the pointed data three 40-minute frames were observed and combined at the RSS file stage to form one two-hour frame. 
Input $\mathrm{F} / 3.4(95 \%)$ or $\mathrm{F} / 3.5(90 \%)$

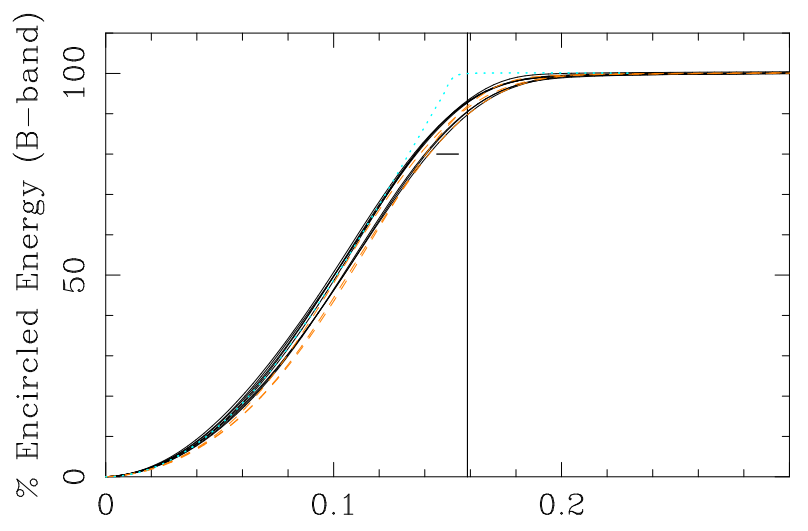

Numerical Aperture

Input $\mathrm{F} / 3.4(95 \%)$ or $\mathrm{F} / 3.5(90 \%)$

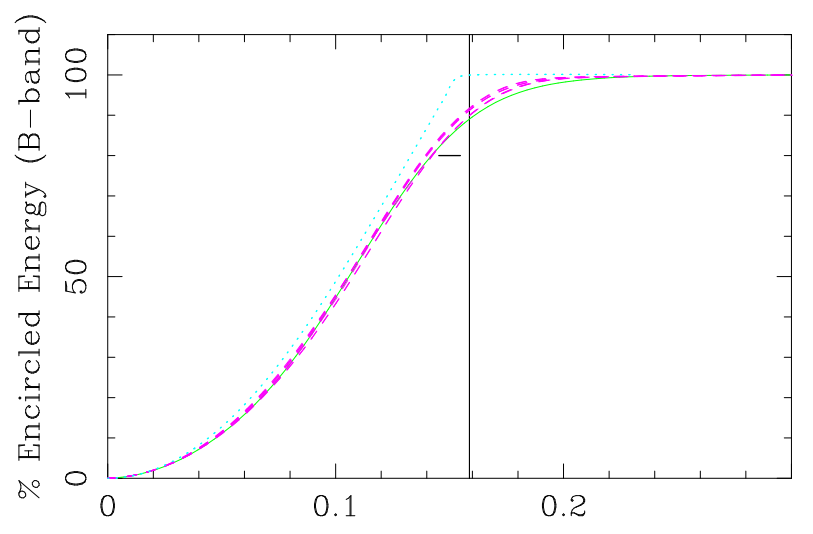

Numerical Aperture
Input $\mathrm{F} / 3.4(95 \%)$ or $\mathrm{F} / 3.5(90 \%)$

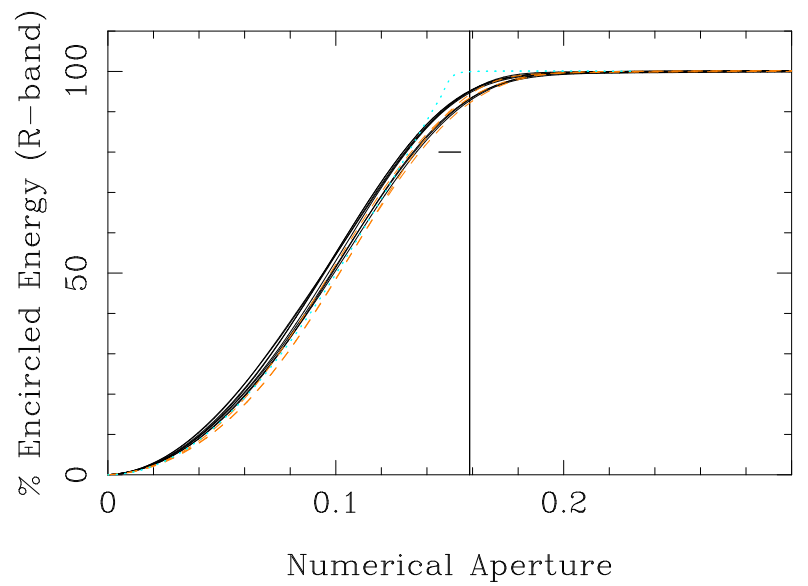

Input $\mathrm{F} / 3.4(95 \%)$ or $\mathrm{F} / 3.5(90 \%)$

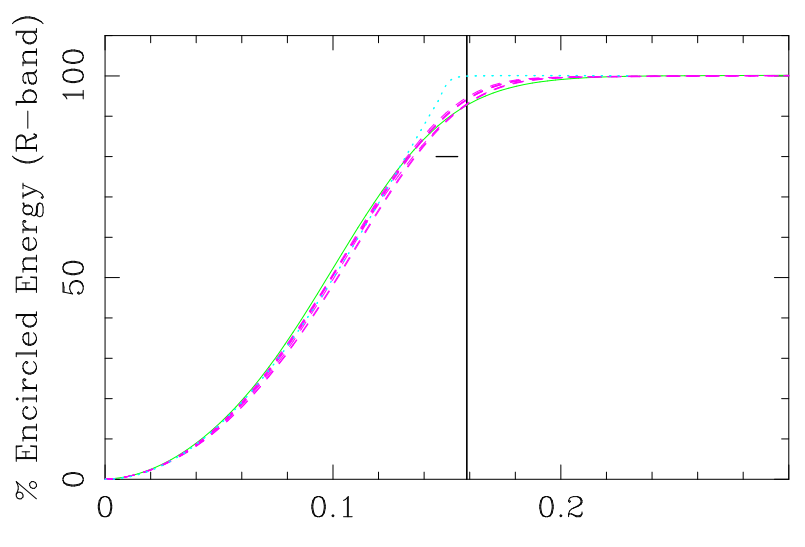

Numerical Aperture

Figure 3. Encircled energy vs NA measured through the blue (4570, left) and red (5960, right) filters. Top row: Comparison between the FRD in a $2.5 \mathrm{~m}$ length of bare AFS105/125Y fibre (dashed orange line) - the same as used in the original SAMI hexabundles - and the FRD from different cores from two hexabundles (solid black lines). At this input f-ratio, the hexabundles have just as good FRD as the bare fibre within errors. Lower row: FRD of $42 \mathrm{~m}$ of the original AFS105/125Y fibre used for SAMI (solid green line), compared to the new fibre chosen for the upgraded SAMI (dashed magenta line) with two different jacket types (acrylate and Tefzel). The replacement fibre in the upgraded SAMI has higher blue throughput (see Table 1), but clearly without sacrificing FRD performance compared to the original fibre used in SAMI. In all plots, the cyan dotted line is the input light encircled energy curve. Measurement errors on this data are illustrated by the horizontal bar. In some cases several cleaves have been done of the single fibres, or several fibres within the bundles and hence the multiple lines of the same type. The similarity between repeated measurements indicates consistent cleaving and little fibre-to-fibre variation within a bundle. The vertical black line marks the $\mathrm{f} / 3.15$ input into AAOmega. 


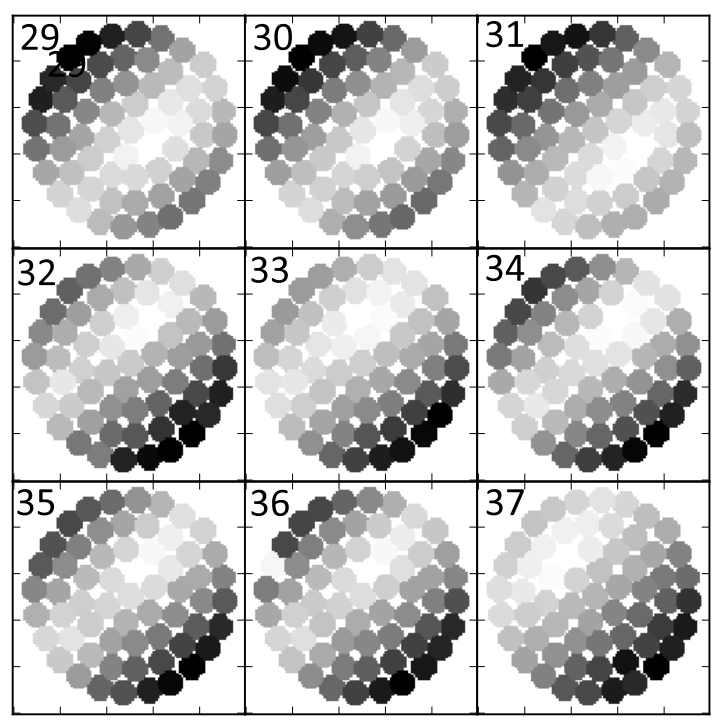

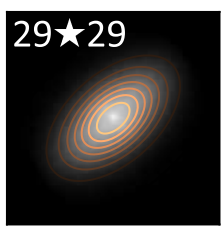
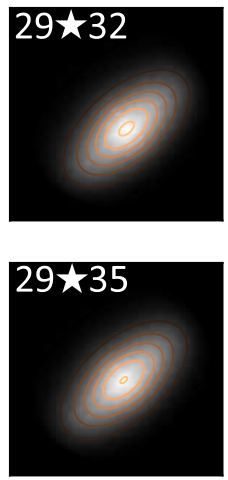
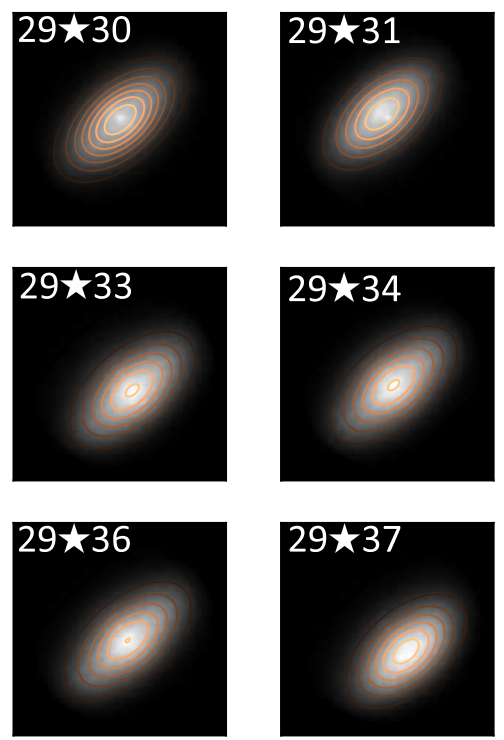

Figure 4. The left-hand panel shows the nine dithered observations of one of the SAMI commissioning galaxies. The images are summed over $6300 \AA$ to $7300 \AA$. The right-hand panel shows the cross-correlation images in grayscale with the two-dimensional Gaussian fits overlaid in contours. These fits are used to determine the offsets to combine the dithered data.

The dithered data could not be combined in RSS format, however, and first had to be reconstructed into full data cubes. This is not trivial as the SAMI spaxels are round and form an irregular grid. To approximate the SAMI sampling on-sky, a super-sampled grid was created at each wavelength slice, with pixels $1 / 10$ th of a core diameter in size. Each SAMI core was then "drawn" as a circle on this super-sampled grid, and filled in with the appropriately scaled flux level. The centres of the cores are determined with an accuracy of $1 / 10$ th of a core diameter. This yields a set of data cubes, with variance cubes created using the same method. Each of the targets had to be treated in this manner.

To combine the dithered data cubes a cross-correlation method was used. Each cube was summed over a large wavelength range, creating something close to a continuum image, with some contamination from emission lines. These continuum frames are then cross-correlated with the first image in the observing sequence to determine the relative offsets in pixel space of each successive image. The offsets are calculated from a two-dimensional Gaussian fit to the cross-correlation functions. This procedure is illustrated in Figure 4 for one of the targets observed with a dither pattern including nine pointings. Figure 4 shows the continuum images and cross-correlation functions and Gaussian fits in the left and right hand panels respectively. The data cubes are then combined using the calculated offsets in pixel space and a median stack.

\subsection{First Science with SAMI}

The SAMI commissioning data included one galaxy field containing 13 targets. One of these proved particularly interesting, and an in depth analysis is presented in Fogarty et al. (in prep.). The continuum and velocity image for this galaxy is shown in Figure 5 and is typical of the data quality for the other galaxies in that run. This galaxy was observed using both the pointed and dithered data and a separate analysis of each data set was performed for comparison. The dithered data was combined using the method outlined above to create two data cubes, one corresponding to the red arm of the spectrograph and the other to the blue. These cubes were then smoothed with a 5-pixel Gaussian and re-binned by a factor of 4 in each spatial axis.

The data analysis was done using custom written python scripts. To analyse the SAMI data cubes, both pointed and dithered, seven individual emission lines were fit and properties were derived from the fits. The lines used are $\mathrm{H} \beta$, [OIII] $\lambda$ 5007, [OI] $\lambda 6300, \mathrm{H} \alpha$, [NII] $\lambda 6583$, [SII] $\lambda 6717$ and [SII] $\lambda 6731$. Figures 6 and 7 shows 

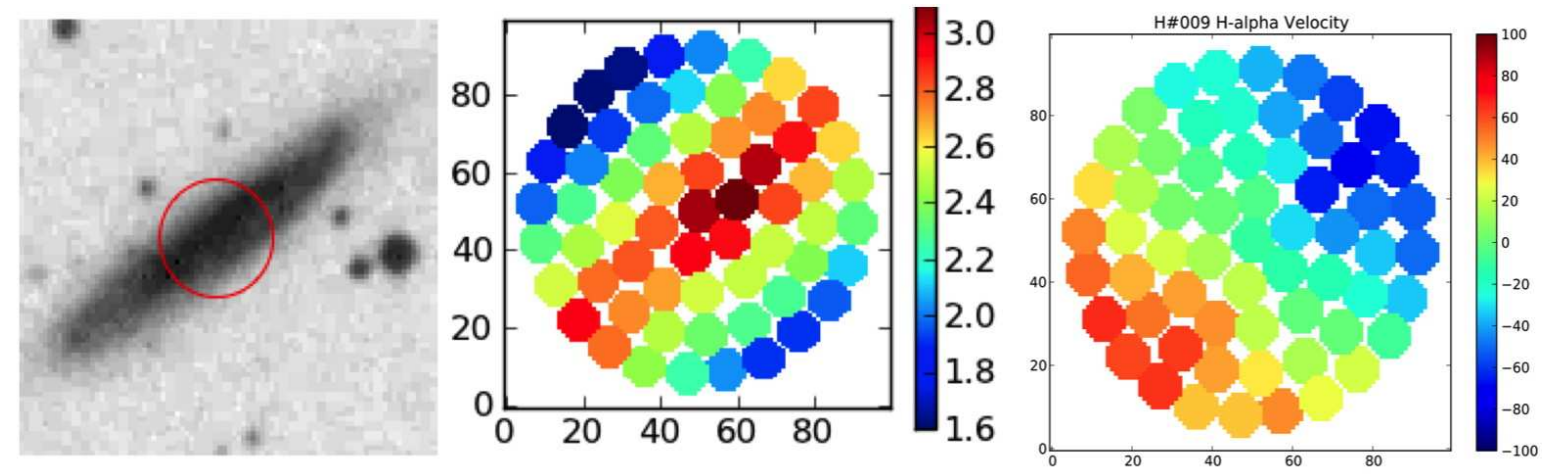

Figure 5. SuperCOSMOS image (left) of one of the galaxies observed with SAMI, followed by the SAMI continuum image (centre) and the SAMI H $\alpha$ velocity image (right). Rotation of the galaxy disk is clearly seen with SAMI. The red circle shows the SAMI bundle size on the SuperCOSMOS image.

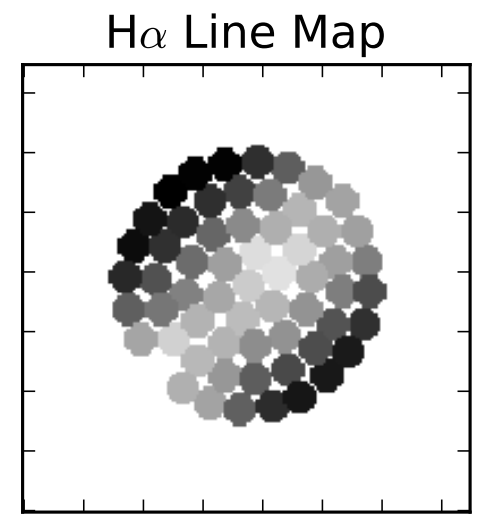

[OI] Line Map

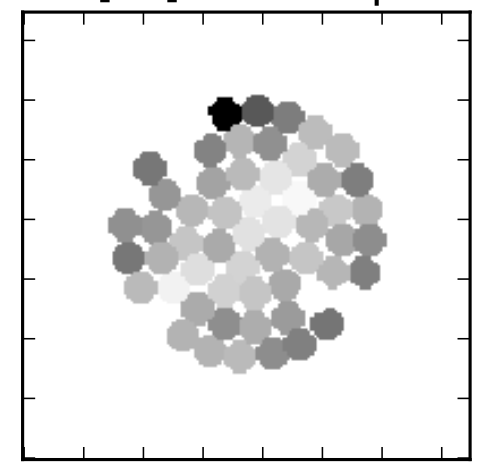

[NII] Line Map

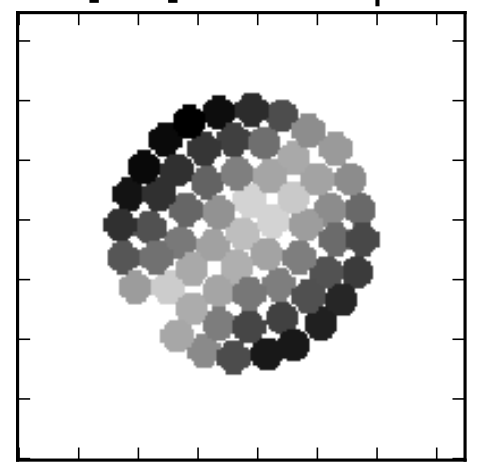

$\mathrm{H} \beta$ Line Map

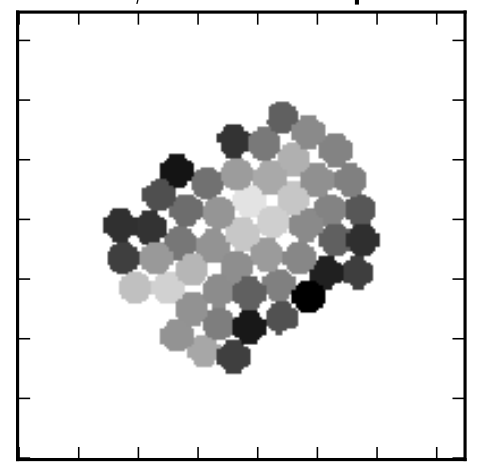

[SII] Line Map

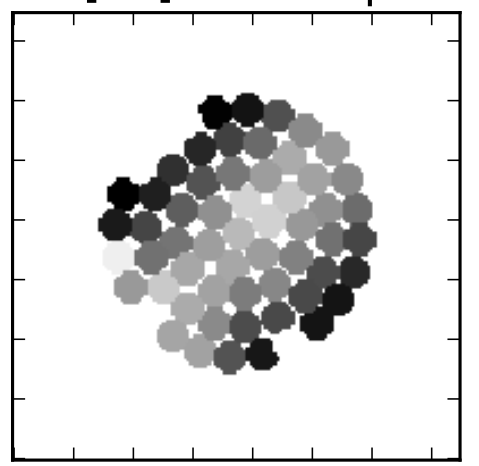

[OIII] Line Map

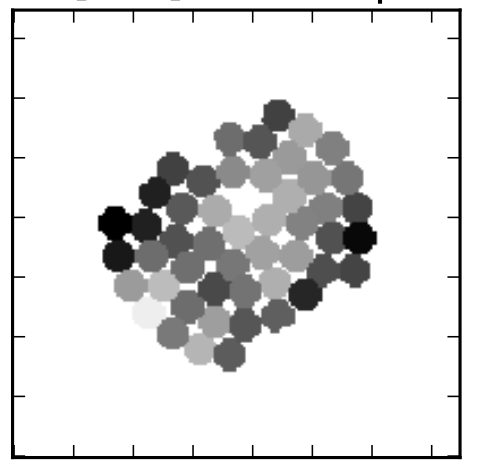

Figure 6. Emission line maps for one of the galaxies in the pointed SAMI commissioning data. Grayscale is in $\log _{10}($ counts $)$ and boxes are 24" on a side. Missing spaxels in the maps are spaxels where the S/N was too low to fit the line.

six line maps derived from the pointed data and the corresponding six from the dithered data. Note that to find the $[\mathrm{SII}]$ line map the $[\mathrm{SII}] \lambda 6717$ and $[\mathrm{SII}] \lambda 6731$ emission features were summed.

Comparing the data it is clear to see the benefits of the dithering technique. The spatial coverage is much more complete and the spatial resolution improved by dithering. For a more in-depth analysis see Fogarty et al. (in prep.). 

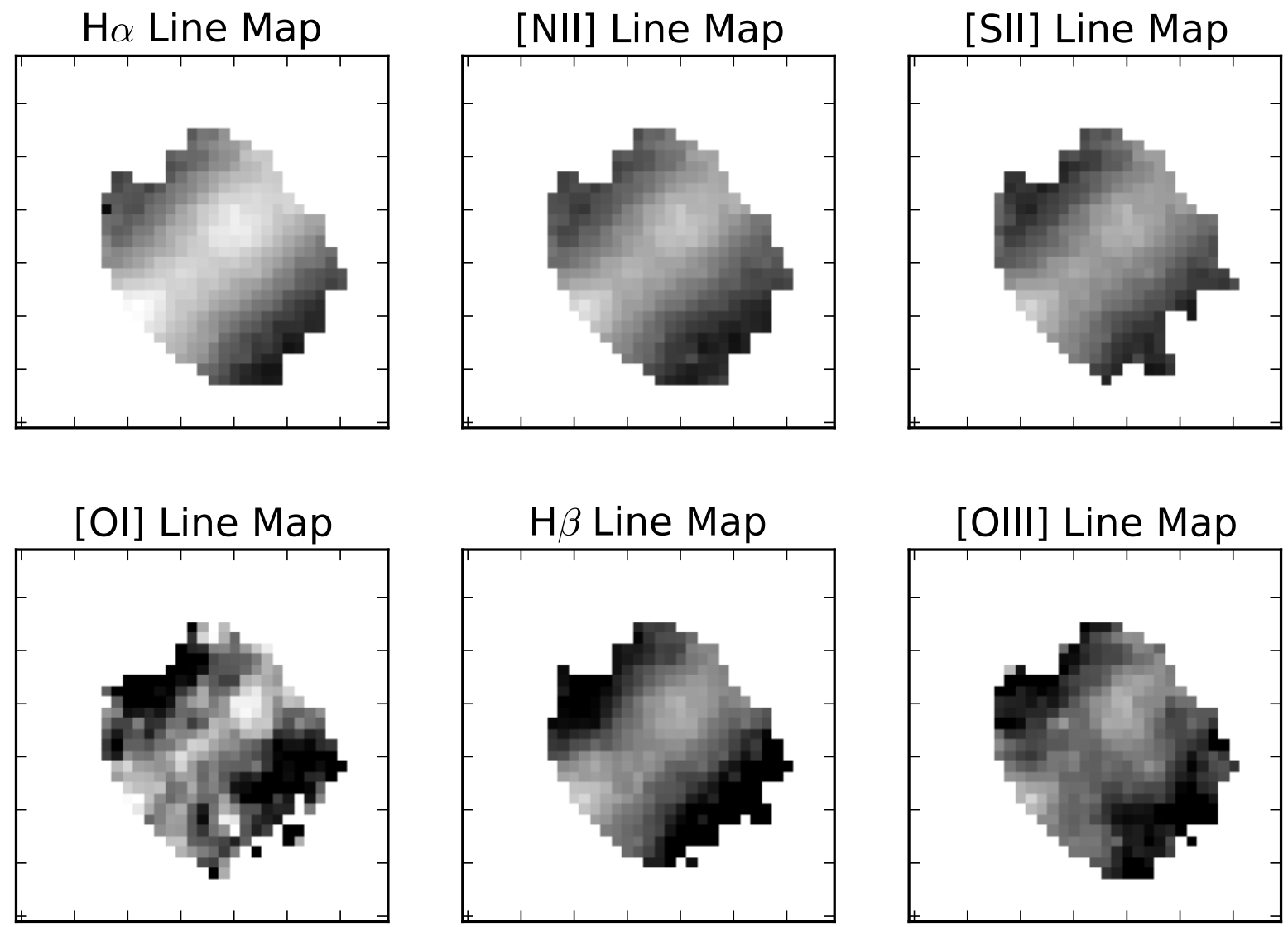

Figure 7. Emission line maps for one of the galaxies in the dithered SAMI commissioning data. Grayscale is in $\log _{10}($ counts $)$ and boxes are $24 "$ on a side. It is clear to see that the spatial coverage is much better, with better sampling and comparably better resolution than the pointed data.

\section{CONCLUSION}

SAMI is the first on-sky instrument to incorporate 'hexabundles' - imaging fibre bundles - and is now operational on the AAT. The FRD performance of the hexabundles has been shown to be as good as that of the same bare fibre, but the original fibre used in SAMI has poorer blue throughput than required. Therefore, the hexabundles and fibre run in SAMI are being replaced by a fibre that has just as good FRD, but with a much higher blue throughput and slightly higher red throughput. The first galaxy results presented here illustrate the power of SAMI to achieve high $\mathrm{S} / \mathrm{N}$ in spatially-resolved spectra of galaxies at $z \leq 0.05$. Dithering techniques have been effectively used to increase the spatial coverage and resolution of the SAMI data. Based on the success of initial data, the SAMI galaxy survey will begin in mid 2012, with an aim to observe several thousand galaxies in the coming years.

\section{ACKNOWLEDGEMENTS}

We warmly thank the staff of the Australian Astronomical Observatory and the Astrophotonics Group at the University of Sydney for their support in making SAMI a reality. JJB is funded by the ARC Centre of Excellence for All-Sky Astrophysics (CAASTRO, CE110001020). JBH and the Astrophotonics group at the University of Sydney is funded by an ARC Federation Fellowship (FF00776384). SMC acknowledges the support of an Australian Research Council (ARC) QEII Fellowship (DP0666615) and an Australian Research Council Future Fellowship (FT100100457). This research was supported by the Australian Research Council Centre of Excellence 
for All-sky Astrophysics (CAASTRO), through project number CE110001020. Thanks to Sam Richards and the SAMI team for the photos of the instrument.

\section{REFERENCES}

[1] Abazajian K. et al., "The First Data Release of the Sloan Digital Sky Survey", AJ, 126, 2081(2003)

[2] Bacon R. et al., "The SAURON project - I. The panoramic integral-field spectrograph", MNRAS, 326, 23 (2001)

[3] Binney J., Nipoti C., Fraternali F., "Do high-velocity clouds form by thermal instability?", MNRAS, 397, $1804(2009)$

[4] Bryant J. J., O’Byrne J. W., Bland-Hawthorn J., Leon-Saval S. G., "Characterisation of hexabundles: initial results", MNRAS 415, 2173 (2011)

[5] Bryant J. J., Fogarty L., Bland-Hawthorn J., Croom S., "Focal ratio degradation in lightly-fused hexabundles", in prep (2012)

[6] Bland-Hawthorn J. et al., "Hexabundles: imaging fiber arrays for low-light astronomical applications" , Optics Express, 19, 2649 (2011)

[7] Bland-Hawthorn J., Sutherland R., Agertz O., Moore B., "The Source of Ionization along the Magellanic Stream", ApJL, 670, L109 (2007)

[8] Blanton M. R., Eisenstein D., Hogg D. W., Schlegel D. J., Brinkmann J., "Relationship between Environment and the Broadband Optical Properties of Galaxies in the Sloan Digital Sky Survey", ApJ, 629, 143 (2005)

[9] Colless M. et al., "The 2dF Galaxy Redshift Survey: spectra and redshifts", MNRAS, 328, 1039 (2001)

[10] Croom S., Saunders W., Heald R., "2DFDR Overhaul", Anglo-Australian Observatory Epping Newsletter, 106, $12(2004)$

[11] Croom S., et al. "The Sydney-AAO Multi-object Integral field spectrograph (SAMI)", MNRAS 421, 872 (2012)

[12] Davis M. et al., "The All-Wavelength Extended Groth Strip International Survey (AEGIS) Data Sets", ApJL, 660, L1 (2007)

[13] Ellison S. L., Patton D. R., Simard L., McConnachie A.W., "Galaxy Pairs in the Sloan Digital Sky Survey. I. Star Formation, Active Galactic Nucleus Fraction, and the Mass-Metallicity Relation", AJ, 135, 1877 (2008)

[14] Fogarty L. et al., in prep, (2012)

[15] Hopkins P. F., Hernquist L., "A Characteristic Division Between the Fueling of Quasars and Seyferts: Five Simple Tests", ApJ, 694, 599 (2009)

[16] Larson R. B., Tinsley B. M., Caldwell C. N., "The evolution of disk galaxies and the origin of S0 galaxies", ApJ, 237, 692 (1980)

[17] Lawrence et al. "Hector: a high-multiplex survey instrument for spatially resolved galaxy spectroscopy", SPIE 8446-195, these proc. (2012)

[18] Le Févre O. et al., "The VIMOS VLT deep survey. First epoch VVDS-deep survey: 11564 spectra with 17.5 IAB 24, and the redshift distribution over 0 z 5", A\&A, 439, 845 (2005)

[19] Lewis I. et al., "The 2dF Galaxy Redshift Survey: the environmental dependence of galaxy star formation rates near clusters", MNRAS, 334, 673 (2002)

[20] Nesvadba N.P.H, Lehnert M.D., De Breuck C., Gilbert A.M., van Breugel W., "Evidence for powerful AGN winds at high redshift: dynamics of galactic outflows in radio galaxies during the Quasar Era", A\&A 491, 407 (2008)

[21] Sancisi R., Fraternali F., Oosterloo T., van der Hulst T., "Cold gas accretion in galaxies", A\&AR, 15, 189 (2008)

[22] Sharp R. et al., "Performance of AAOmega: the AAT multi-purpose fiber-fed spectrograph", Proc. SPIE 6269, 62690G (2006)

[23] Sharp R., Birchall M. N., "Optimal Extraction of Fibre Optic Spectroscopy", PASA, 27, 91 (2010)

[24] Tremaine S. et al., "The Slope of the Black Hole Mass versus Velocity Dispersion Correlation", ApJ, 574, $740(2002)$

[25] Tody, D., "The IRAF Data Reduction and Analysis System", Proc. SPIE 627, 733 (1986) 